\title{
Role of fimV in type II secretion system-dependent protein secretion of Pseudomonas aeruginosa on solid medium
}

\author{
Correspondence \\ Romé Voulhoux \\ voulhoux@ifr88.cnrs-mrs.fr
}

Received 30 September 2010

Revised 2 March 2011

Accepted 22 April 2011

\author{
Gérard P. F. Michel, Anthony Aguzzi, Geneviève Ball, Chantal Soscia, \\ Sophie Bleves and Romé Voulhoux
}

\begin{abstract}
Laboratoire d'Ingénierie des Systèmes Macromoléculaires (LISM-UPR9027), CNRS and AixMarseille Université, Institut de Microbiologie de la Méditerranée, 31 Chemin Joseph Aiguier, 13402 Marseille Cedex 20, France
\end{abstract}

\begin{abstract}
Although classical type II secretion systems (T2SSs) are widely present in Gram-negative bacteria, atypical T2SSs can be found in some species. In Pseudomonas aeruginosa, in addition to the classical T2SS Xcp, it was reported that two genes, $x p h A$ and $x q h A$, located outside the $x c p$ locus were organized in an operon $(\mathrm{PaQa})$ which encodes the orphan $\mathrm{PaOa}$ subunit. This subunit is able to associate with other components of the classical Xcp machinery to form a functional hybrid T2SS. In the present study, using a transcriptional lacZ fusion, we found that the $\mathrm{PaQ} a$ operon was more efficiently expressed (i) on solid LB agar than in liquid LB medium, (ii) at $25^{\circ} \mathrm{C}$ than at $37^{\circ} \mathrm{C}$ and (iii) at an early stage of growth. These results suggested an adaptation of the hybrid system to particular environmental conditions. Transposon mutagenesis led to the finding that vfr and fimV genes are required for optimal expression of the orphan PaQa operon in the defined growth conditions used. Using an original culturing device designed to monitor secretion on solid medium, the ring-plate system, we found that T2SS-dependent secretion of exoproteins, namely the elastase LasB, was affected in a fimV deletion mutant. Our findings led to the discovery of an interplay between FimV and the global regulator Vfr triggering the modulation of the level of Vfr and consequently the modulation of T2SS-dependent secretion on solid medium.
\end{abstract}

\section{INTRODUCTION}

The Gram-negative pathogen Pseudomonas aeruginosa has evolved a number of sophisticated mechanisms that help it to battle hostile environment and host defences. One of these mechanisms involves secretion of virulence factors such as proteases, phospholipases and toxins that constitute efficient molecular weapons allowing the colonization of host cells by bacteria (Bleves et al., 2010). Six secretion systems have been described so far in Gram-negative bacteria ranging from the type I to the type VI secretion system. $P$. aeruginosa possesses five of the six secretion systems reported, with only the type IV secretion system absent in this bacterium. Such a diverse arsenal shows that it can be a powerful pathogen involved in a wide range of severe and sometimes fatal diseases including cystic fibrosis, nosocomial pneumonia and infections in surgery patients (Bodey et al., 1983).

Most of the main degradative enzymes of $P$. aeruginosa are secreted by the type II secretion system (T2SS), a two-step mechanism involving a transient periplasmic step before

Abbreviations: OS, quorum sensing; T2SS, type II secretion system. exoenzymes are targeted to the extracellular medium. This system is genetically organized into two divergent operons encoding XcpR-Z and XcpPQ components. The T2SS can be viewed as a molecular nanomachine that consists of an inner-membrane platform, including XcpPRSYZ proteins, and a fibrillar structure (the pseudopilus) composed of the pseudopilins XcpTUVWX that act like a piston using elongation and retraction movements to push exoproteins out of the cell through a channel made by multimers of the secretin XcpQ (Michel \& Voulhoux, 2009). The T2SS process was shown to be very specific since components of the machinery cannot be exchanged between distant species (Bouley et al., 2001; Filloux, 2004). In other respects, exoproteins cannot be secreted from one species to another, except for closely related species (de Groot et al., 2001). Such a high specificity was shown to be particularly dependent on XcpP and XcpQ proteins and homologues, which are the gate keepers of the T2SS machinery (Lindeberg et al., 1996; Bleves et al., 1999) and constitute a functional subunit referred as the PQ subunit in $P$. aeruginosa.

Outside the $x c p$ locus components of the classical T2SS, we previously reported the presence of two orphan 
genes, $x p h A$ and $x q h A$, organized in an operon, which were found to be homologous to the $x c p P$ and $x c p Q$ genes, respectively. These genes encode the orphan $\mathrm{PaQa}$ subunit which can replace the classical PQ subunit and associate with the XcpR-Z components to make up a functional hybrid T2SS machinery (Michel et al., 2007). The hybrid T2SS was shown to efficiently secrete all the T2SSdependent exoproteins, except for the aminopeptidase PaAP (PA2939) which accumulated within the cells.

Although the role of this atypical system has not yet been elucidated, we hypothesized that it could (i) either co-exist with the classical T2SS or serve as a rescue system, (ii) only be required in some specific growth conditions such as growth on solid media or in biofilms or (iii) be induced in response to unknown signals occurring during host infection (Michel et al., 2007). In the present work, we aimed to investigate the growth conditions required to optimize functioning of the hybrid T2SS and finding regulators that control expression of the genes encoding the orphan $\mathrm{PaQ}$ a subunit, in order to better understand the physiological role of this atypical secretory system.

\section{METHODS}

Bacterial strains, plasmids and growth conditions. Bacterial strains and plasmids are listed in Table 1 . P. aeruginosa strains were grown either in Luria-Bertani (LB) or tryptic soy broth (TSB) at the adequate growth temperature. When required, 150 or $300 \mu \mathrm{g}$ carbenicillin $\mathrm{ml}^{-1}$ (in liquid or solid culture, respectively) and $75 \mu \mathrm{g}$ gentamicin $\mathrm{ml}^{-1}$ were added to the growth medium. To generate the fimV deletion, $500 \mathrm{bp}$ upstream and $500 \mathrm{bp}$ downstream of the fimV gene were amplified by overlapping PCR using dfimV1, dfimV2, dfimV3 and dfimV4 primers (Table 1) and the PCR product was cloned in pKNG101 giving the mutator vector $\mathrm{pKNG} \Delta$ fimV. The $x c p Q$ $x q h A$ deletion was generated using $\mathrm{pKNG} \Delta x q h A$ mutation vector in the PAO $1 \Delta \mathrm{Q}$ strain. The resulting strain PAO1 $\Delta \mathrm{QQ}$ a was then deleted for fimV by using pKNG $\Delta$ fimV resulting in the PAO1 $\Delta \mathrm{QQa} \Delta$ fimV strain. The deletion mutants were generated as described by Bleves et al. (1999). Wild-type fimV gene obtained from plasmid pASM281 was subcloned at the KpnI/XbaI sites of pMMB67EH.

Transcriptional fusions. Transcriptional $\mathrm{PPaQa}-\mathrm{lac} Z$ (formerly called xphA-lacZ in Michel et al., 2007) and PPQ-lacZ fusions were generated in wild-type PAO1 and in mutants by following the procedure of Hoang et al. (2000). Expression of the transcriptional fusions was assayed by $\beta$-galactosidase activity (Sambrook et al., 1989) using cells grown either in liquid or on solid LB media. In order to standardize growth on solid medium, $2 \mathrm{ml}$ liquid LB medium was first inoculated with frozen cells and grown for $8-10 \mathrm{~h}$ at $37^{\circ} \mathrm{C}$. Then, $0.1 \mathrm{OD}_{600}$ equivalent cell unit was loaded on LB agar plates, rigorously streaked in squares of $1.5 \times 1.5 \mathrm{~cm}$ and incubated at the required temperature for $15 \mathrm{~h}$. Cells were scraped from the plates, resuspended in $1 \mathrm{ml} \mathrm{LB}$ and $1 \mathrm{OD}_{600}$ equivalent cell unit was centrifuged $\left(10000 \mathrm{~g}, 5 \mathrm{~min}, 4{ }^{\circ} \mathrm{C}\right) . \beta$-Galactosidase activity was assayed at $30^{\circ} \mathrm{C}$ as described by Sambrook et al. (1989) and results were expressed as described by Miller (1972). Statistical analysis of data was performed by paired Student's $t$-test (Excel software).

Construction of mutant library. A mutant library was generated in the strain bearing the $\mathrm{P} P a Q a-l a c Z$ transcriptional fusion using the transposon Mariner C9 according to the procedure of Kulasekara et al. (2005). The library was screened on selective LB agar medium containing $75 \mu \mathrm{g}$ gentamicin $\mathrm{ml}^{-1}, 25 \mu \mathrm{g}$ Irgasan $\mathrm{ml}^{-1}$ and $25 \mu \mathrm{g} \mathrm{X}$ $\mathrm{Gal} \mathrm{ml}^{-1}$. Localization of the transposon insertions was carried out by two-round arbitrary PCR using pBT20-1/ARB1D and pBT20-2/ARB2A primer pairs (Table 1). PCR products were sequenced (GATC Company) and mutated genes were identified by BLAST analysis with the sequenced genome of $P$. aeruginosa (http://v2.pseudomonas.com).

Protein secretion on solid medium. Protein secretion on solid medium was investigated using the ring-plate culturing system adapted from Levin et al. (2007) and modified as follows. Cells were cultured essentially as described above for the study of transcriptional fusions. Cells $\left(0.2 \mathrm{OD}_{600}\right.$ equivalent cell unit) were added on an LB agar disc (1.5-2 $\mathrm{mm}$ thick) loaded on a polycarbonate filter $(0.05 \mu \mathrm{m})$ placed at the centre of the ring-plate. The filter membrane was upheld by the ring compartment of the ring-plate and was in contact with the LB liquid medium filling the rings at the bottom of the device. The ring-plate was then incubated at the required temperature for 5-20 h according to the experiment. During growth, secreted proteins crossed both the agar disc and the filter and were finally recovered in the liquid compartment, while cells were retained by the filter. The liquid filtrate containing extracellular proteins was either directly used for protease activity assays or precipitated on ice with $10 \%(\mathrm{w} / \mathrm{v})$ trichloroacetic acid (TCA) and the proteins were treated as previously described for SDS-PAGE or immunodetection analyses (Michel et al., 2007).

Protein analysis and immunodetection. Extracellular proteins were separated from cells by centrifugation $\left(10000 \mathrm{~g}, 5 \mathrm{~min}, 4{ }^{\circ} \mathrm{C}\right)$. Extracellular proteins were precipitated on ice with $10 \%(\mathrm{w} / \mathrm{v})$ TCA and treated as described previously (Michel et al., 2007). Cellular and extracellular proteins were solubilized with Laemmli lysis buffer (Laemmli, 1970), separated by SDS-PAGE (12\% w/v) and visualized by either immunodetection as described previously (Michel et al., 2007) or Imperial blue protein stain (Pierce) and silver staining (Swain \& Ross, 1995). When indicated, protein quantification was performed using ImageJ software (http://www.rsbweb.nih.gov/ij/).

Proteolytic activities. Protease activity from liquid extracts was assayed according to Wretlind \& Pavlovskis (1984) using Azocasein as substrate. Protease activities on solid medium were regularly detected after streaking cells on tryptic soy agar (TSA) containing skimmed milk and incubating plates at 37 or $25{ }^{\circ} \mathrm{C}$. Secretion of active proteases was detected as a clear halo.

\section{RESULTS AND DISCUSSION}

\section{Proteolytic activities dependent on hybrid T2SS}

As previously reported, reconstitution of the hybrid T2SS constituted by the XcpR-Z components of the classical T2SS and the orphan subunit PaQa restored the secretion of most of the T2SS substrates (Michel et al., 2007). In the present study, using a proteolytic activity assay, a secretion test commonly used in secretion studies, we observed that the $\triangle \mathrm{PQPaQa}$ mutant complemented for the $\mathrm{PaQa}$ mutation exhibited only about $40 \%$ of the total extracellular proteolytic activity recovered in the parent strain (Table 2). These results suggested that restoration of the secretion efficiency by the hybrid T2SS was only partial in liquid cultures. In contrast, the complemented mutant grown on a TSA-milk plate, another typical secretion assay, exhibited the same hydrolytic halo as the parent strain, showing that in these growth conditions, protein secretion was fully restored (Table 2). These results are consistent 
Table 1. Strains, plasmids and primers

\begin{tabular}{|c|c|c|}
\hline Strain/plasmid/primer & Description/sequence & Reference or source \\
\hline \multicolumn{3}{|l|}{ Strains } \\
\hline \multicolumn{3}{|l|}{ Escherichia coli } \\
\hline 1047 & met supE hsdS recA & Laboratory collection \\
\hline CC118 ( $\lambda$ pir) & $\Delta($ ara-leu $)$ araD $\Delta$ lacX74 galE galK phoA-20 thi-1 rpsE rpoB Arg(Am) recAl, $\mathrm{Rf}^{\mathrm{r}}(\lambda$ pir) & Herrero et al. (1990) \\
\hline SM10 ( $\lambda$ pir) & tri thr leu tonA lacY SupE recA:: RP4-2-Tc:: Mu Km${ }^{\mathrm{r}} \lambda$ pir & Herrero et al. (1990) \\
\hline $\mathrm{PAO} 1$ & Protrotroph, chl-2 & B. Holloway \\
\hline $\mathrm{PAO} 1 \Delta \mathrm{Q}$ & PAO1 strain with the $x c p Q$ gene deleted & de Groot et al. (2001) \\
\hline $\mathrm{PAO} 1 \Delta \mathrm{PaQa}$ & PAO1 strain with the $x p h A$ and $x q h A$ genes deleted & Michel et al. (2007) \\
\hline $\mathrm{PAO} 1 \triangle \mathrm{PQPaQa}$ & PAO1 strain with the $x c p P, x c p Q, x p h A$ and $x q h A$ genes deleted & Michel et al. (2007) \\
\hline $\mathrm{PAO} 1:: \mathrm{PPaQa}-\mathrm{lacZ}$ & $\mathrm{PAO} 1$ strain with transcriptional $\mathrm{P} P a Q a-l a c Z$ fusion & Michel et al. (2007) \\
\hline PAO1 : : PPQ-lacZ & $\mathrm{PAO} 1$ strain with transcriptional $\mathrm{P} P Q-$ lac $Z$ fusion & This study \\
\hline $\mathrm{PAO} 1 \Delta \mathrm{Q} \Delta$ fim $V$ & $\mathrm{PAO} 1 \Delta \mathrm{Q}$ mutant with the fim $V$ gene deleted & This study \\
\hline \multicolumn{3}{|l|}{ Plasmids } \\
\hline pRK2013 & $\mathrm{Km}^{\mathrm{r}}, \mathrm{ColE} 1, \mathrm{Tra}^{+} \mathrm{Mob}^{+}$ & Figurski \& Helinski (1979) \\
\hline pKNG101 & $\mathrm{Sm}^{\mathrm{r}}$, oriR6K mobRK2 sabBR ${ }^{+}$(suicide vector) & Kaniga et al. (1991) \\
\hline pKNG $\Delta \mathrm{PaQa}$ & Mutator plasmid for $x p h A-x q h A$ deletion & Michel et al. (2007) \\
\hline $\mathrm{pKNG} \Delta$ fim $V$ & Mutator plasmid for fim $V$ deletion & This study \\
\hline Mini CTX-lacZ & $\mathrm{Tc}^{\mathrm{r}}, \Omega-F R T$-attP-MCS, ori int oriT & Hoang et al. (2000) \\
\hline Mini CTX-PPaQa-lacZ & Mini CTX bearing transcriptional fusion $x p h A^{\prime}-l a c Z$ & Michel et al. (2007) \\
\hline Mini CTX-PPQ-lacZ & Mini CTX bearing transcriptional fusion $x c p P^{\prime}-l a c Z$ & This study \\
\hline pFLP2 & $\mathrm{Ap}^{\mathrm{r}}$, ori1600 oriT rep $c I_{857} F L P$ sacB & Hoang et al. (2000) \\
\hline pBT20 & $\mathrm{Ap}^{\mathrm{r}}, \mathrm{Gm}^{\mathrm{r}}, \mathrm{P}$ tac oriRK6K oriT, Mariner C9 & Kulasekara et al. (2005) \\
\hline pMMB-PaQa & pMMB190 bearing $x p h A-x q h A$ genes & Michel et al. (2007) \\
\hline pSW205 & $\mathrm{Ap}^{\mathrm{r}}$, lac $Z$ fusion vector & Gambello et al. (1993) \\
\hline pTS400 & $\mathrm{Ap}^{\mathrm{r}}$, lasB'-lacZ translational fusion in pSW205 & Passador et al. (1993) \\
\hline \multicolumn{3}{|l|}{ Oligonucleotides } \\
\hline dfimV1 & 5'-AGCGTATCGGACCGTTGAACGTTACCT-3' & This study \\
\hline dfimV2 & 5'-TTCATTTCCGGATCACATAGTGTAATCCCT-3' & This study \\
\hline dfimV3 & 5'-AGGGATTACACTATGTGATCCGGAAATGAA-3' & This study \\
\hline dfimV4 & 5'-ATGGGTGCCGACCAGTACCTGGGCCGC-3' & This study \\
\hline pBT20-1 & 5'-AAGCTGTGGTATGGCTGTGCA-3' & Liberati et al. (2006) \\
\hline ARB1D & 5'-GGCCAGGCCTGCAGATGATGNNNNNNNNNNGTAT-3' & Liberati et al. (2006) \\
\hline pBT20-2 & 5'-AATTGTGAGCGGATAACAATTT-3' & Liberati et al. (2006) \\
\hline ARB2A & 5'-GGCCAGGCCTGCAGATGATG-3' & Liberati et al. (2006) \\
\hline
\end{tabular}

with those published previously (Michel et al., 2007). Thus, it can be speculated that culture conditions could affect production and/or secretion efficiency of proteolytic exoenzymes. Indeed, the differences observed could be related to a higher efficiency of the hybrid T2SS during growth on solid medium compared with liquid medium, an activation of some of the proteases secreted by the hybrid T2SS or by the secretion of yet unknown proteases. These hypotheses drove us, as a first step, to study the effect of growth conditions on the expression of the PaQa operon.

\section{Expression of the PaQa operon during growth on solid medium}

In order to compare expression levels of the $P a Q a$ operon in cells grown in liquid and solid media, we used the 
Table 2. Extracellular proteolytic activity and expression of $\mathrm{PPaQa}-\mathrm{lac} Z$ fusion in liquid and solid cultures

Strains were grown in either liquid or solid LB media at $37{ }^{\circ} \mathrm{C}$ in the presence of carbenicillin. IPTG ( $2 \mathrm{mM}$ final concentration) was added as inducer in order to produce the orphan subunit PaQa. Images of the proteolytic assay on TSA milk plates are shown in column three.

\begin{tabular}{|lcc|}
\hline Strain & \multicolumn{2}{c|}{ Proteolytic activity on: } \\
\cline { 2 - 3 } & $\begin{array}{c}\text { Liquid LB } \\
\text { medium }\end{array}$ & Solid medium \\
\hline $\mathrm{PAO} 1 / \mathrm{pMMB}$ & 100 & \\
& \\
$\mathrm{PAO} 1 / \mathrm{pPaQa}$ & $109 \pm 7.5$ & \\
& \\
\hline $\mathrm{PQPaQa} / \mathrm{pMMB}$ & $14.3 \pm 3.6$ & \\
& \\
\hline $\mathrm{PQPaQa} / \mathrm{pPaQa}$ & $36.8 \pm 1.5$ & \\
& \\
\end{tabular}

*Total proteolytic activity from LB liquid cultures assayed using Azocasein as substrate. Results are expressed (\%) relative to the parent strain bearing the empty vector pMMB190, and are the mean \pm SEM of two independent experiments.

transcriptional $\mathrm{P} P a Q a-l a c Z$ fusion already generated in our lab in the wild-type strain PAO1 as a tool (Michel et al., 2007). As reported previously, the activity of the fusion was found to be relatively low and constant throughout growth in liquid medium (Michel et al., 2007) in contrast with the increased expression of $\mathrm{P} P Q-$ lac Z and $\mathrm{PRZ}$-lacZ transcriptional fusions (data not shown and Chapon-Hervé et al., 1997). Here, we found that expression of the orphan operon was threefold higher in cells grown on solid LB agar $(302.8 \pm 15.3$ Miller units) than in cells grown in liquid LB $(99.3 \pm 12$ Miller units). This difference was significant compared with the control PAO1 strain $(P<0.001$ by Student's $t$-test). This result, in agreement with our hypothesis, suggested that the orphan subunit, and thus the hybrid T2SS, could be particularly adapted to growth on solid medium. However, using the transcriptional fusions PPQ-lacZ (monitoring expression of the operon encoding the homologous classical PQ subunit) and PRZlacZ (monitoring expression of the other T2SS genes) as controls, we found that, similar to the $P a Q a$ operon, the $P Q$ operon was also activated to a greater level on solid medium (3.8-fold) compared with liquid medium. These results could be expected considering that classical and orphan subunits can be exchanged and play the same function in the T2SS secretory machinery. In contrast, the $R Z$ operon was almost the same (1.3-fold more than liquid medium) on solid medium (data not shown) showing that not all promoters are similarly activated on solid medium.

\section{Effect of growth temperature and cell ageing on expression of $\mathrm{PaQa}$}

Recently, using transcriptomic and 2D-PAGE analyses, we reported that gene expression and secretome profiles of $P$. aeruginosa were altered when cells were grown at $25{ }^{\circ} \mathrm{C}$ instead of $37^{\circ} \mathrm{C}$ (Termine \& Michel, 2009). These results suggested that growth temperature may also alter a number of processes related to protein secretion. Therefore, we studied the effect of temperature on the expression of the transcriptional $\mathrm{PPaQa}-$ lacZ fusion in cells grown on solid medium at various growth temperatures. As shown in Fig. 1(a), expression of the orphan operon correlated inversely with the temperature; the activity of the transcriptional fusion decreased progressively from 25 to $37^{\circ} \mathrm{C}$. In contrast, the activity of the transcriptional $\mathrm{P} P Q-$ lac $Z$ fusion monitoring expression of the operon encoding the classical PQ subunit (homologous to the orphan subunit) increased from 25 to $37{ }^{\circ} \mathrm{C}$, suggesting a better adaptation to growth at $37{ }^{\circ} \mathrm{C}$. However, it should be emphasized that the transcription level of the $P P Q-l a c Z$ fusion at $25{ }^{\circ} \mathrm{C}$ is comparable to that of the $\mathrm{pPaQa}-\mathrm{lac} Z$ fusion. Thus, it seems likely that hybrid and classical T2SSs could co-exist at the lower growth temperature.

In order to further define the optimal conditions of expression for the orphan genes, the wild-type strain expressing the $\mathrm{PPaQa-lacZ}$ fusion was grown on solid LB medium at $25{ }^{\circ} \mathrm{C}$ and activity of the fusion was assayed for $\beta$-galactosidase activity throughout growth. The optimal activity was obtained between 8 and 15 h of growth and decreased after longer incubation (Fig. 1b). This result suggested that the orphan unit could most probably be useful for secretion at an early stage of growth, as proposed previously (Michel et al., 2007).

Therefore, several interesting features can be drawn from our results. (i) The orphan subunit could be particularly dedicated to suboptimal growth temperature, whereas the classical one is better adapted to higher temperatures. (ii) Optimal expression of the orphan operon occurs at an early stage of the bacterial growth. (iii) Orphan and classical subunits can co-exist during growth at suboptimal temperatures but their functioning could be adapted to some specific environmental conditions and controlled by different regulatory mechanisms. From these observations, it can be proposed that the orphan subunit might play an 
(a)

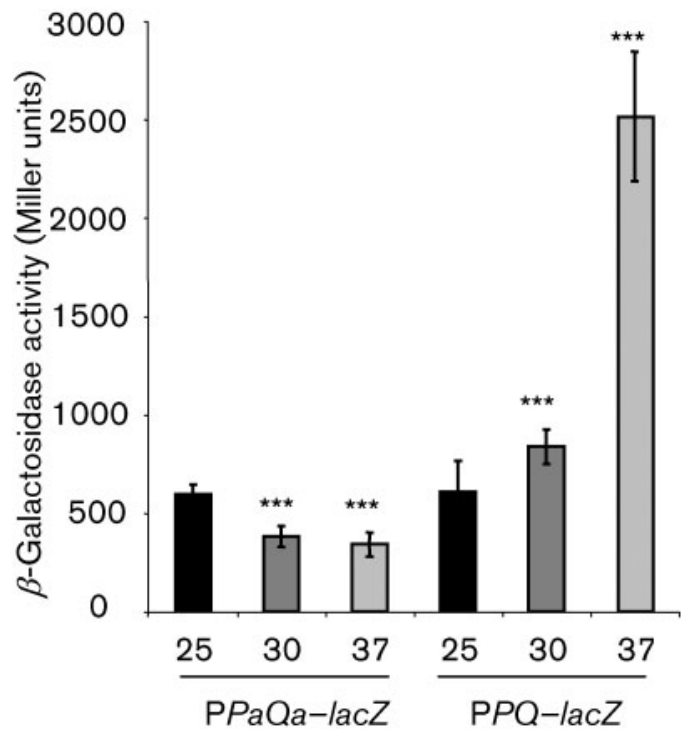

(b)

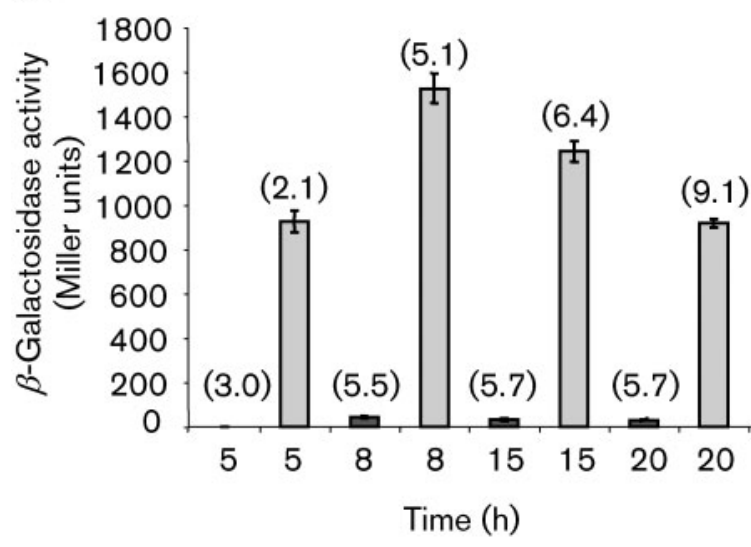

Fig. 1. Effect of growth temperature and cell ageing on the expression of $P a Q a$ and $P Q$ operons. (a) PAO1 : PPaQa-lac $Z$ and $P A O 1$ : : PPQ-lac Z strains were inoculated on solid $L B$ medium as described in Methods, and incubated for $15 \mathrm{~h}$ at 25,30 or $37{ }^{\circ} \mathrm{C}$. $\beta$-Galactosidase activities were assayed using $1 \mathrm{OD}_{600}$ cell equivalent. Results are mean \pm SEM of three independent experiments. Significant difference compared with growth at $25^{\circ} \mathrm{C}$ is indicated by asterisks ( ${ }^{\star \star *} P<0.001$, Student's $t$-test). (b) The PAO1::PPaQa-lacZ strain was grown at $25^{\circ} \mathrm{C}$ on solid LB medium and cells were withdrawn from the agar plates at the indicated times. The $\mathrm{OD}_{600}$ of each culture, calculated as described in Methods, is indicated above each bar. $\beta$-Galactosidase activities were assayed using $1 \mathrm{OD}_{600}$ cell equivalent. Light grey bars, $\mathrm{PPaOa-lacZ}$ fusion; dark grey bars, lac $Z$ promoter-less fusion control. Results are mean \pm SEM of three independent experiments.

important role in the environment and not in the host, and could contribute to the establishment of $P$. aeruginosa as a nosocomial pathogen on solid surfaces, for instance in hospitals, while the classical subunit could be more dedicated to direct human infection.
Genes involved in the regulation of expression of the PaQa operon

Results obtained from transcriptional studies suggested that expression of the genes encoding the orphan subunit could be under a specific regulatory control. Indeed, our results suggest a different regulatory control of $\mathrm{PaQ} a$ and $P Q$ operons, since the latter is known to be regulated by quorum sensing (QS) (Chapon-Hervé et al., 1997; Schuster et al., 2003; Wagner et al., 2003). To identify PaQa regulators, a mutant library was engineered by using the properties of the transposon Mariner C9. This transposon was introduced into the wild-type strain bearing the transcriptional $\mathrm{P} P a Q a-l a c Z$ fusion, and recombinants were screened on X-Gal plates according to the intensity of blue staining. Seven clones, less blue than the control cells, were further investigated since they could represent mutants affected in putative activators. Transposon insertions in strains were localized by arbitrary PCR amplification and sequencing of the PCR product. Mutations affected PA1370 and PA1547 (hypothetical proteins), PA1549 (predicted ATPase involved in copper transport), PA1554 (cytochrome oxidase), PA0652 (Vfr) and finally PA3115 (FimV). Two different clones containing a transposon insertion at two distinct positions were mutated in the $v f r$ gene. These results are in agreement with microarray studies carried out by Wolfgang et al. (2003) showing that expression of $x p h A$ and $x q h A$ genes was highly decreased in a vfr mutant.

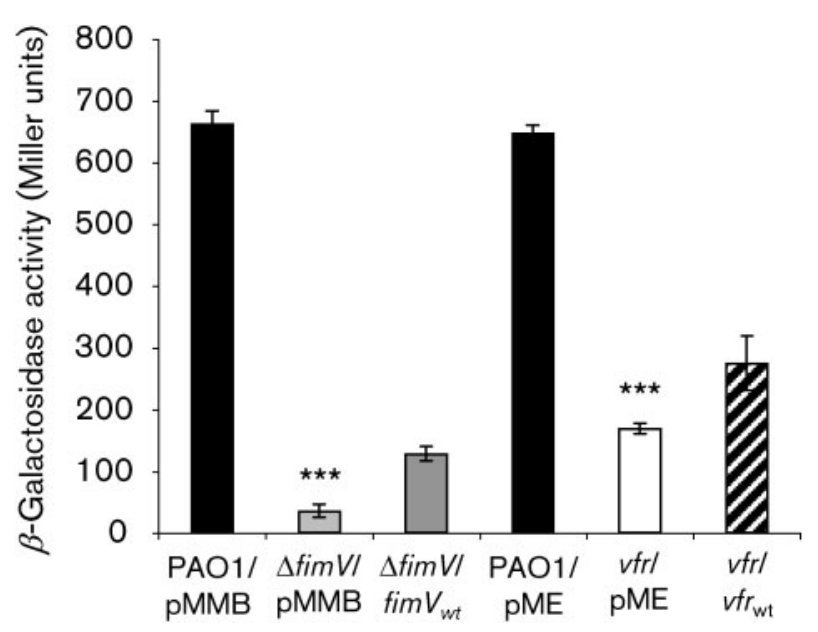

Fig. 2. Influence of fimV and vfr genes on the expression of the $\mathrm{PaQa}$ operon. $\beta$-Galactosidase activity of the PPaOa-lacZ fusion was assayed in fimV and vfr mutants and their complemented counterparts grown on LB agar. Results are mean \pm SEM of two independent experiments. Significant difference compared with the control PAO1 strain is indicated by asterisks $\left({ }^{\star \star \star} P<0.001\right.$, Student's $t$-test). 


\section{Effect of vfr and fim $V$ mutations on the expression of the PaQa operon}

We focused on $v$ fr and fim $V$ genes already reported in the literature. The former gene encodes a well-known global regulator that controls several processes (expression of genes encoding proteases, exotoxin A, twitching motility and QS) (Albus et al., 1997; Beatson et al., 2002; Suh et al., 2002; Kanack et al., 2006; Fox et al., 2008) and the latter encodes a protein involved in type IV pili assembly but has been poorly studied so far (Semmler et al., 2000; Ahn et al., 2004). Both genes were also reported to be required for twitching motility and indeed, as expected, we observed that the selected mutants were impaired for this movement mode (data not shown). As shown in Fig. 2, activity of the $\mathrm{PPaQa}-\mathrm{lacZ}$ fusion was reduced by 18 -fold in the fimV deletion mutant and less drastically in the $v f r$ transposon mutant (fourfold). Complementation of the mutations increased activity of the fusions in these mutants, by 3.5fold for fimV and 1.6-fold for vfr. This partial complementation was most probably due to the non-physiological expression level of $f i m V$ and $v f r$ from plasmids. Nevertheless, our results showed that $v f r$ and $\operatorname{fim} V$ genes are clearly (directly or indirectly) involved in the process that controls expression of the PaQa operon.

\section{Effect of the fim $V$ deletion on T2SS secretion on solid medium}

Having defined the experimental conditions required to optimize expression of the orphan subunit, we investigated protein secretion on solid medium using an original culturing technique adapted for $P$. aeruginosa cells from Levin et al. (2007): the ring-plate system. This culturing system was found to be functional, as the secretome of the PAO1 strain exhibited a similar exoprotein profile to that obtained with a classical liquid culture (Fig. 3a, lanes 1 and 2 ). As the first attempts to detect exoproteins specifically secreted by the hybrid T2SS on ring-plates using a $\triangle \mathrm{PaQa}$ mutant were unsuccessful, a new approach was investigated. Since we demonstrated a direct relationship between fim $V$ and expression of the genes encoding the $\mathrm{PaQa}$ subunit, it was tempting to speculate that protein substrates of the hybrid T2SS might be detected by comparing secretome profiles of the parent strain PAO1 and its $\Delta$ fim $V$ isogenic mutant. However, no exoprotein

(a)

\section{Liquid culture Ring-plate cultures}

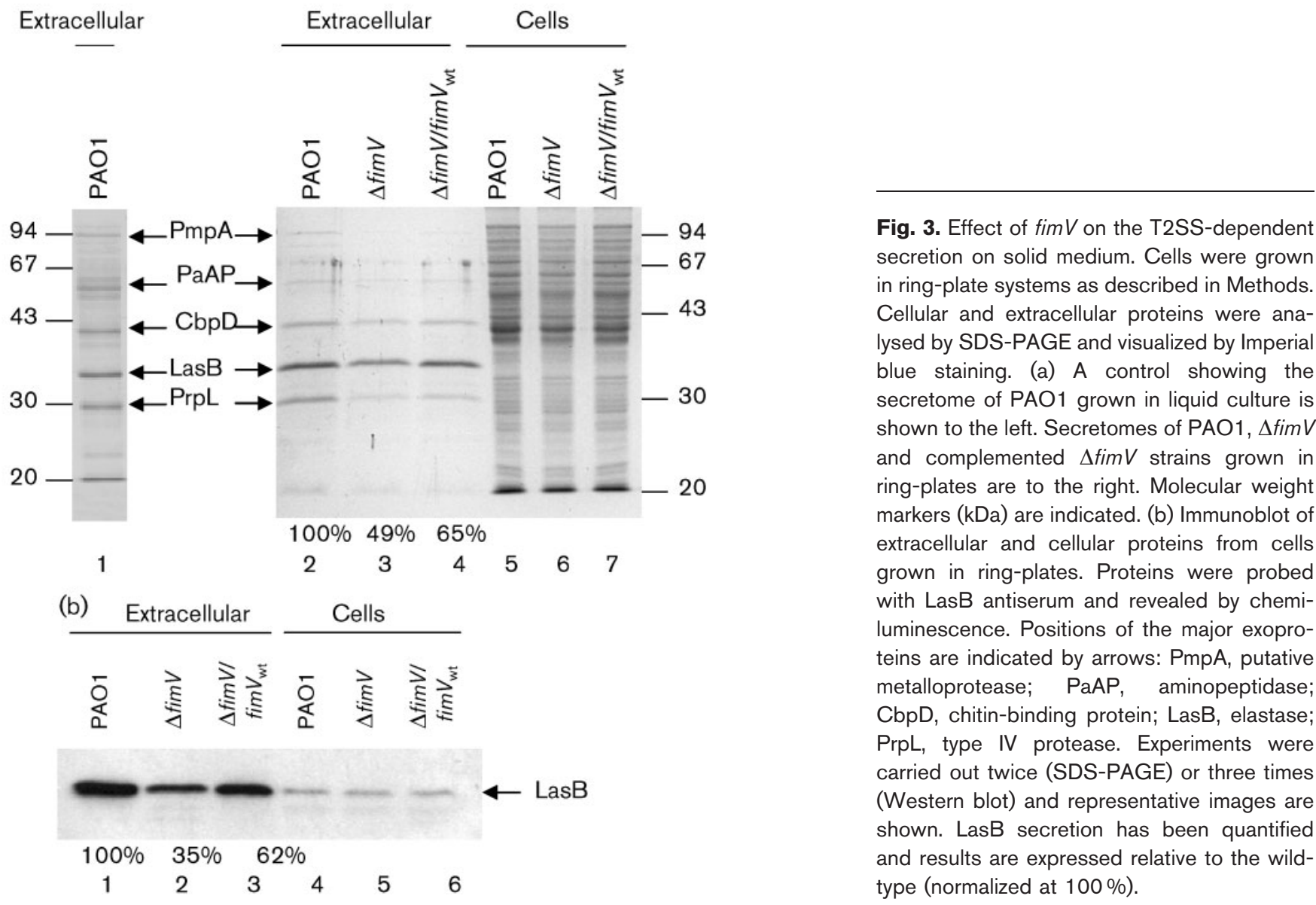


specific for the hybrid T2SS could be detected in these comparative studies (Fig. 3a, lanes 2 and 3). Interestingly, deletion of the fim $V$ gene caused a slight decrease in all the major exoproteins identified in the secretome of PAO1, and complementation of the mutation partially restored the secretion efficiency (Fig. 3a). Indeed, immunodetection of the major exoprotease secreted by PAO1, the elastase LasB, confirmed that the amount of protein was decreased in the fim $V$ mutant (Fig. 3b, lane 2) and its level was partially restored to that of the parent strain after complementation of the mutation (Fig. 3b, lane 3). These results suggested that fim $V$ could affect secretion via not only the hybrid T2SS but also the classical T2SS. LasB was not found to be accumulated in cells (Fig. 3b, lanes 4-6) in agreement with the cellular protein profiles observed in Fig. 3 (a) (lanes 5-7). Furthermore, $P$. aeruginosa mutant strains with the $x c p Q$ gene deleted (encoding the secretin of the Xcp machinery, the classical T2SS) retained a residual hydrolytic activity shown on TSA-milk plates (Martínez et al., 1998 and Fig. 4a). This activity is related to the hybrid secretion system functioning with the orphan subunit $\mathrm{PaQ}$. Deletion of both $x c p Q$ and $x q h A$ ( $\triangle \mathrm{QQ}$ a mutant) led to the complete disappearance of the proteolytic halo. We speculated that since the $\operatorname{fim} V$ gene is required for optimal

(a)

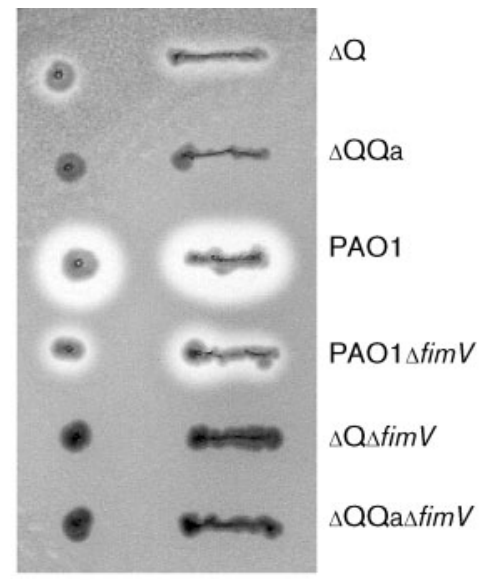

(b)

Extracellular
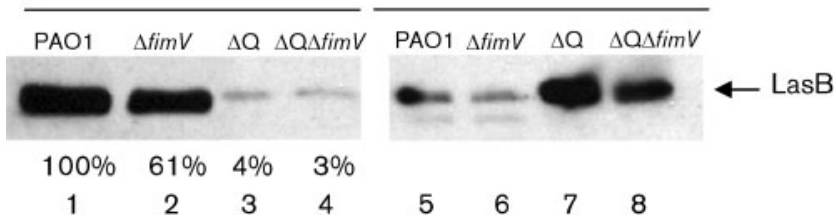

Fig. 4. Influence of fim $V$ deletion on the hybrid T2SS secretion and on LasB production on solid medium. (a) Cells were either pricked or streaked on TSA-milk plates and their proteolytic activity was evaluated according to the size of the clear halo zone observed on the plate. (b) Immunoblot of extracellular and cellular proteins from cells grown in ring-plates. Proteins probed with LasB antiserum were detected by chemiluminescence. This experiment was performed twice. LasB secretion has been quantified and the results are expressed relative to the wild-type (normalized at $100 \%$ ). expression of the $x p h A-x q h A$ operon, the PaQa-dependent residual hydrolytic activity should be abolished or at least drastically decreased in a mutant with a deletion in both the $x c p Q$ and $\operatorname{fim} V$ genes. To this end, we engineered fim $V$ mutants in $x c p Q$ and $x c p Q / x q h A$ backgrounds and tested them for proteolytic activity. As shown in Fig. 4(a) and as expected, the double mutant $\Delta \mathrm{Q} \Delta \operatorname{fim} V$ was devoid of a proteolytic halo and displayed the same behaviour as the $\Delta$ QQa mutant. This result suggests that fim $V$ was required for secretion of proteases, mainly of the major exoprotease LasB, by the hybrid system. Immunodetection of LasB confirmed that as already shown in Fig. 3(b), the extracellular LasB level was decreased in PAO1 $\Delta$ fimV (Fig. 4b, lane 2). This decreased level of LasB could also explain the small hydrolytic halo observed for the $\mathrm{PAO} 1 \Delta \mathrm{fim} V$ strain in the protease assay on the plate (Fig. 4a). As expected, LasB was faintly or not detected in extracellular extracts of the $\Delta \mathrm{Q}$ and $\Delta \mathrm{Q} \Delta$ fim $V$ mutants (Fig. 4b, lanes 3 and 4), but both strains accumulated large amounts of LasB within the cells showing that they are impaired for LasB secretion (Fig. 4b, lanes 7 and 8).

\section{Influence of fim $V$ on lasB expression}

In contrast with the results obtained with $\Delta \mathrm{Q}$ and $\Delta \mathrm{Q} \Delta$ fim $V$ mutant strains, intracellular accumulation of LasB was not observed in the PAO1 $\Delta$ fimV mutant (Fig. 3 b, lane 5, and Fig. 4b, lane 6). These results could suggest that either intracellularly accumulated LasB was degraded by proteases in this mutant strain or lasB expression rather than secretion as such was affected by the fim $V$ deletion. In line with the last hypothesis, a translational lasB-lac $Z$ fusion expressed in the wild-type PAO1 strain and in the fimV mutant showed that activity of the fusion was decreased by $70 \%$ in the mutant strain (Fig. 5a). Therefore, it can be concluded that FimV is directly or indirectly required for efficient control of las $B$ expression. Since FimV is thought to be involved in type IV pili assembly, we hypothesized that blocking this process could trigger perturbations of the cell envelope and sensing signals, leading to an alteration of more global regulatory mechanisms, particularly those involved in las $B$ expression. las $B$ expression is known to be dependent on $\mathrm{Vfr}$ control via QS regulation (Gambello \& Iglewski, 1991; Albus et al., 1997). Moreover, as indicated in Fig. 2, the global regulator Vfr was found to be an activator of the expression of orphan genes that encode the PaQa subunit. In order to test a possible relationship between FimV and the Vfr protein, we checked the level of $v f r$ expression in fim $V$ mutants. Cellular proteins from PAO1, PAOl $\Delta$ fimV, $\Delta \mathrm{Q}$ and $\Delta \mathrm{Q} \Delta$ fim $V$ strains were analysed by Western blotting and probed with Vfr antiserum. Immunodetection revealed that the Vfr level decreased in fimV mutants compared with wild-type or $\Delta \mathrm{Q}$ parent strains (Fig. 5b, c). The relationship between FimV and Vfr appeared to be tight since complementation of the fimV mutation fully restored the Vfr level to that of the parent strain (Fig. 5c, lane 3). Thus, these results show that high amounts of $\mathrm{Vfr}$ are required for optimal expression of lasB. Moreover, it should be pointed out that the level of Vfr 


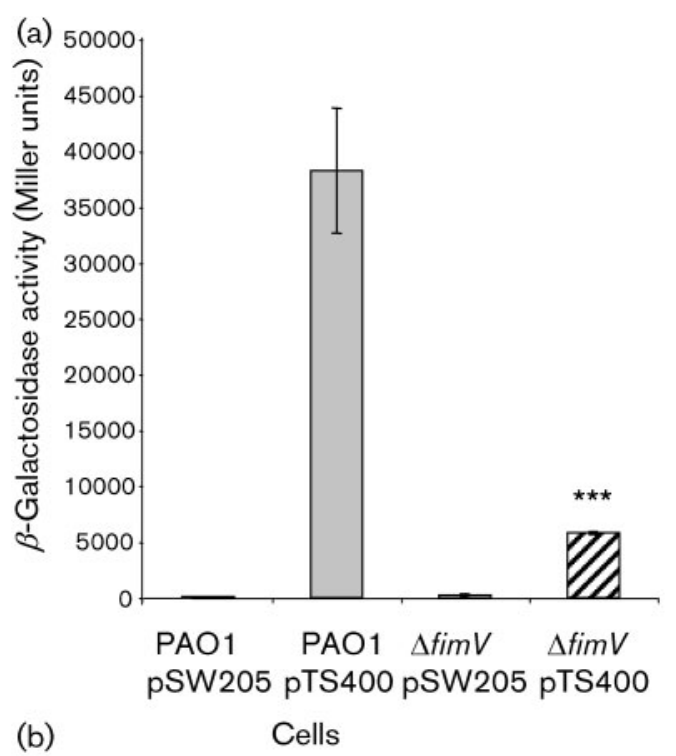

(b)

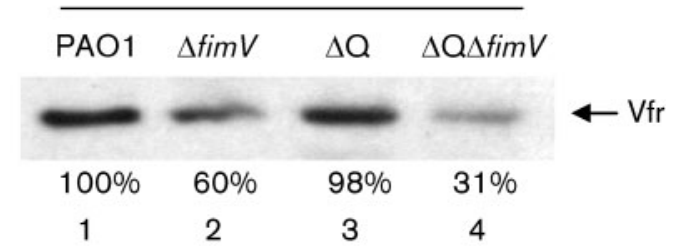

(c)

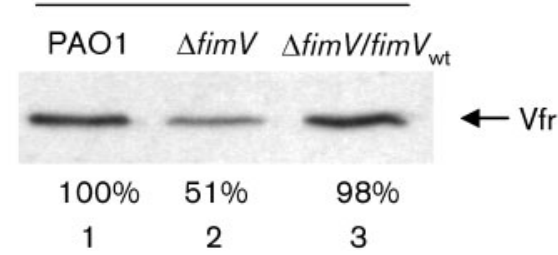

Fig. 5. Factors regulating las $B$ expression. (a) Activity of the las $B-$ lacZ translational fusion from PAO1 and its cognate fimV mutant grown on solid LB medium. pSW205, empty vector; pTS400, vector bearing the translational las $B-l a c Z$ fusion. Results are mean \pm SEM of two independent experiments. Significant difference compared with the control PAO1 strain is indicated by asterisks $\left({ }^{* \star} P<0.001\right.$ in the Student's $t$-test). (b and c) Effect of fimV deletion on Vfr levels. Cellular proteins obtained from PAO1, $\Delta \mathrm{Q}$ and their cognate fimV mutants were analysed by immunoblotting. Proteins were probed with $\mathrm{Vfr}$ antiserum and detected by chemiluminescence. Results are representative of two independent experiments. The same protein extracts as those shown in Fig. 4(b) (b) or Fig. 3(b) (c) were used here. The amount of Vfr has been quantified and the results are expressed relative to the wildtype (normalized at $100 \%$ ).

is not altered in the $\Delta \mathrm{Q}$ mutant impaired for classical T2SS, suggesting a specific link between the absence of FimV and the decrease in Vfr.

In contrast with Vfr, little is known about the FimV protein of $P$. aeruginosa. Nevertheless, it has been reported recently that a fimV mutant was defective in cAMP synthesis (Fulcher et al., 2010) and that cAMP was required for Vfr activity and $v f r$ expression (Fuchs et al., 2010). Vfr has also been shown to control expression of the lasR promoter and thus QS in a cAMP-independent manner (Fuchs et al., 2010). However, in contrast with our results, none of these studies indicated a direct relationship between FimV and Vfr proteins.

FimV protein is predicted to contain a LysM domain (http://v2.pseudomonas.com and http://pfam.sanger.ac. uk) which is thought to have a general peptidoglycan binding function and to be present in a variety of enzymes involved in bacterial cell degradation (Interpro entry IPR018392). As FimV has been shown to be involved in type IV pilus assembly (Semmler et al., 2000), one could imagine that the loss of FimV affects a peptidoglycan breaching step necessary for pilus assembly. It could also be possible that the same peptidoglycan breaching mechanism is required for assembly of the T2SS machinery. Furthermore, in our experimental conditions, the absence of FimV could also cause a particular perturbation of the envelope due to defective pilus assembly and lead to an alteration of sensing signals involved in regulatory mechanisms such as Vfr control. This hypothesis is also supported by the studies of Baysse et al. (2005) showing that membrane alterations can modulate QS in P. aeruginosa.

\section{Conclusions}

In summary, our results showed that high levels of Vfr are required for optimal expression of genes encoding the orphan subunit of the hybrid T2SS and LasB. Although unexpected, FimV was shown to interact with Vfr by a mechanism that acts as a modulator of the Vfr level. Such fine tuning could also modulate both the level of Vfrdependent exoproteins and the efficiency of hybrid and classical T2SS according to the physiological state of the bacteria. Although no substrate specific for the hybrid T2SS has been identified so far, our results offer new insights into the function of this atypical secretion system. At the current stage of our investigation, it can be suggested that the hybrid T2SS can at least serve as a rescue system in growth conditions where the PQ subunit of the classical T2SS is affected or is poorly efficient. However, it can be reasonably claimed that in contrast with the classical T2SS, the hybrid T2SS could play a particular role in defined environmental conditions such as growth on solid medium at suboptimal growth temperature and at a relatively low cell density. Further work is now needed to improve the ring-plate culturing system in order to identify potential substrates of the hybrid T2SS of $P$. aeruginosa grown on solid medium and to elucidate the physiological role of this atypical secretion system.

\section{ACKNOWLEDGEMENTS}

The authors are grateful to Professor Han Wösten for his expert advice and for kindly providing us with a model ring-plate device, Caroline Chambon and Abdelrahim Zoued for their participation 
during their training, John Mattick's lab for providing us with pASM281 plasmid, Barbara Iglewski's lab for providing us with pSW205 and pTS400 plasmids, Katrina Forest for her generous gift of Vfr antiserum, Christophe Bordi for helpul discussions on transposon mutagenesis and Steve Garvis for reading the manuscript and helpful discussions. This work was supported by CNRS and by Agence Nationale de la Recherche Program 'Jeune Chercheur' grant ANRJC07-183230, 3D-Pilus.

\section{REFERENCES}

Ahn, K. S., Ha, U., Jia, J., Wu, D. \& Jin, S. (2004). The truA gene of Pseudomonas aeruginosa is required for the expression of type III secretory genes. Microbiology 150, 539-547.

Albus, A. M., Pesci, E. C., Runyen-Janecky, L. J., West, S. E. \& Iglewski, B. H. (1997). Vfr controls quorum sensing in Pseudomonas aeruginosa. J Bacteriol 179, 3928-3935.

Baysse, C., Cullinane, M., Dénervaud, V., Burrowes, E., Dow, J. M., Morrissey, J. P., Tam, L., Trevors, J. T. \& O'Gara, F. (2005). Modulation of quorum sensing in Pseudomonas aeruginosa through alteration of membrane properties. Microbiology 151, 2529-2542.

Beatson, S. A., Whitchurch, C. B., Sargent, J. L., Levesque, R. C. \& Mattick, J. S. (2002). Differential regulation of twitching motility and elastase production by Vfr in Pseudomonas aeruginosa. J Bacteriol 184, 3605-3613.

Bleves, S., Gérard-Vincent, M., Lazdunski, A. \& Filloux, A. (1999). Structure-function analysis of XcpP, a component involved in general secretory pathway-dependent protein secretion in Pseudomonas aeruginosa. J Bacteriol 181, 4012-4019.

Bleves, S., Viarre, V., Salacha, R., Michel, G. P. F., Filloux, A. \& Voulhoux, R. (2010). Protein secretion systems in Pseudomonas aeruginosa: a wealth of pathogenic weapons. Int J Med Microbiol 300, 534-543.

Bodey, G. P., Bolivar, R., Fainstein, V. \& Jadeja, L. (1983). Infections caused by Pseudomonas aeruginosa. Rev Infect Dis 5, 279-313.

Bouley, J., Condemine, G. \& Shevchik, V. E. (2001). The PDZ domain of OutC and the $\mathrm{N}$-terminal region of OutD determine the secretion specificity of the type II out pathway of Erwinia chrysanthemi. J Mol Biol 308, 205-219.

Chapon-Hervé, V., Akrim, M., Latifi, A., Williams, P., Lazdunski, A. \& Bally, M. (1997). Regulation of the xcp secretion pathway by multiple quorum-sensing modulons in Pseudomonas aeruginosa. Mol Microbiol 24, 1169-1178.

de Groot, A., Koster, M., Gérard-Vincent, M., Gerritse, G., Lazdunski, A., Tommassen, J. \& Filloux, A. (2001). Exchange of Xcp (Gsp) secretion machineries between Pseudomonas aeruginosa and Pseudomonas alcaligenes: species specificity unrelated to substrate recognition. J Bacteriol 183, 959-967.

Figurski, D. H. \& Helinski, D. R. (1979). Replication of an origincontaining derivative of plasmid RK2 dependent on a plasmid function provided in trans. Proc Natl Acad Sci U S A 76, 1648-1652.

Filloux, A. (2004). The underlying mechanisms of type II protein secretion. Biochim Biophys Acta 1694, 163-179.

Fox, A., Haas, D., Reimmann, C., Heeb, S., Filloux, A. \& Voulhoux, R. (2008). Emergence of secretion-defective sublines of Pseudomonas aeruginosa PAO1 resulting from spontaneous mutations in the $v f r$ global regulatory gene. Appl Environ Microbiol 74, 1902-1908.

Fuchs, E. L., Brutinel, E. D., Jones, A. K., Fulcher, N. B., Urbanowski, M. L., Yahr, T. L. \& Wolfgang, M. C. (2010). The Pseudomonas aeruginosa $\mathrm{Vfr}$ regulator controls global virulence factor expression through cyclic AMP-dependent and -independent mechanisms. J Bacteriol 192, 3553-3564.
Fulcher, N. B., Holliday, P. M., Klem, E., Cann, M. J. \& Wolfgang, M. C. (2010). The Pseudomonas aeruginosa Chp chemosensory system regulates intracellular cAMP levels by modulating adenylate cyclase activity. Mol Microbiol 76, 889-904.

Fürste, J. P., Pansegrau, W., Frank, R., Blöcker, H., Scholz, P., Bagdasarian, M. \& Lanka, E. (1986). Molecular cloning of the plasmid RP4 primase region in a multi-host-range tacP expression vector. Gene 48, 119-131.

Gambello, M. J. \& Iglewski, B. H. (1991). Cloning and characterization of the Pseudomonas aeruginosa lasR gene, a transcriptional activator of elastase expression. J Bacteriol 173, 3000-3009.

Gambello, M. J., Kaye, S. \& Iglewski, B. H. (1993). LasR of Pseudomonas aeruginosa is a transcriptional activator of the alkaline protease gene (apr) and an enhancer of exotoxin A expression. Infect Immun 61, 1180-1184.

Heeb, S., Blumer, C. \& Haas, D. (2002). Regulatory RNA as mediator in GacA/RsmA-dependent global control of exoproduct formation in Pseudomonas fluorescens CHA0. J Bacteriol 184, 1046-1056.

Herrero, M., de Lorenzo, V. \& Timmis, K. N. (1990). Transposon vectors containing non-antibiotic resistance selection markers for cloning and stable chromosomal insertion of foreign genes in Gramnegative bacteria. J Bacteriol 172, 6557-6567.

Hoang, T. T., Kutchma, A. J., Becher, A. \& Schweizer, H. P. (2000). Integration-proficient plasmids for Pseudomonas aeruginosa: sitespecific integration and use for engineering of reporter and expression strains. Plasmid 43, 59-72.

Kanack, K. J., Runyen-Janecky, L. J., Ferrell, E. P., Suh, S. J. \& West, S. E. (2006). Characterization of DNA-binding specificity and analysis of binding sites of the Pseudomonas aeruginosa global regulator, Vfr, a homologue of the Escherichia coli cAMP receptor protein. Microbiology 152, 3485-3496.

Kaniga, K., Delor, I. \& Cornelis, G. R. (1991). A wide-host-range suicide vector for improving reverse genetics in gram-negative bacteria: inactivation of the blaA gene of Yersinia enterocolitica. Gene 109, 137-141.

Kulasekara, H. D., Ventre, I., Kulasekara, B. R., Lazdunski, A., Filloux, A. \& Lory, S. (2005). A novel two-component system controls the expression of Pseudomonas aeruginosa fimbrial cup genes. Mol Microbiol 55, 368-380.

Laemmli, U. K. (1970). Cleavage of structural proteins during the assembly of the head of bacteriophage T4. Nature 227, 680-685.

Levin, A. M., de Vries, R. P. \& Wösten, H. A. (2007). Localization of protein secretion in fungal colonies using a novel culturing technique; the ring-plate system. J Microbiol Methods 69, 399-401.

Liberati, N. T., Urbach, J. M., Miyata, S., Lee, D. G., Drenkard, E., Wu, G., Villanueva, J., Wei, T. \& Ausubel, F. M. (2006). An ordered, nonredundant library of Pseudomonas aeruginosa strain PA14 transposon insertion mutants. Proc Natl Acad Sci U S A 103, 28332838.

Lindeberg, M., Salmond, G. P. \& Collmer, A. (1996). Complementation of deletion mutations in a cloned functional cluster of Erwinia chrysanthemi out genes with Erwinia carotovora out homologues reveals OutC and OutD as candidate gatekeepers of species-specific secretion of proteins via the type II pathway. Mol Microbiol 20, 175190.

Martínez, A., Ostrovsky, P. \& Nunn, D. N. (1998). Identification of an additional member of the secretin superfamily of proteins in Pseudomonas aeruginosa that is able to function in type II protein secretion. Mol Microbiol 28, 1235-1246.

Michel, G. P. \& Voulhoux, R. (2009). The type II secretory system (T2SS) in Gram negative bacteria: a molecular nanomachine for secretion of Sec and Tat-dependent extracellular proteins. In Bacterial 
Secreted Proteins, vol. 1, pp. 67-92. Edited by K. Wooldridge. Norfolk: Caister Academic Press.

Michel, G. P., Durand, E. \& Filloux, A. (2007). XphA/XqhA, a novel GspCD subunit for type II secretion in Pseudomonas aeruginosa. J Bacteriol 189, 3776-3783.

Miller, J. H. (1972). Experiments in Molecular Genetics. Cold Spring Harbor, NY: Cold Spring Harbor Laboratory.

Morales, V. M., Bäckman, A. \& Bagdasarian, M. (1991). A series of wide-host-range low-copy-number vectors that allow direct screening for recombinants. Gene 97, 39-47.

Passador, L., Cook, J. M., Gambello, M. J., Rust, L. \& Iglewski, B. H. (1993). Expression of Pseudomonas aeruginosa virulence genes requires cell-to-cell communication. Science 260, 1127-1130.

Sambrook, J., Fritsch, E. F. \& Maniatis, T. (1989). Molecular Cloning: a Laboratory Manual, 2nd edn. Cold Spring Harbor, NY: Cold Spring Harbor Laboratory.

Schuster, M., Lostroh, C. P., Ogi, T. \& Greenberg, E. P. (2003). Identification, timing, and signal specificity of Pseudomonas aeruginosa quorum-controlled genes: a transcriptome analysis. J Bacteriol 185, 2066-2079.

Semmler, A. B., Whitchurch, C. B., Leech, A. J. \& Mattick, J. S. (2000). Identification of a novel gene, fim $V$, involved in twitching motility in Pseudomonas aeruginosa. Microbiology 146, 1321-1332.
Suh, S. J., Runyen-Janecky, L. J., Maleniak, T. C., Hager, P., MacGregor, C. H., Zielinski-Mozny, N. A., Phibbs, P. V., Jr \& West, S. E. (2002). Effect of $v f r$ mutation on global gene expression and catabolite repression control of Pseudomonas aeruginosa. Microbiology 148, 1561-1569.

Swain, M. \& Ross, N. W. (1995). A silver stain protocol for proteins yielding high resolution and transparent background in sodium dodecyl sulfate-polyacrylamide gels. Electrophoresis 16, 948-951.

Termine, E. \& Michel, G. P. (2009). Transcriptome and secretome analyses of the adaptive response of Pseudomonas aeruginosa to suboptimal growth temperature. Int Microbiol 12, 7-12.

Wagner, V. E., Bushnell, D., Passador, L., Brooks, A. I. \& Iglewski, B. H. (2003). Microarray analysis of Pseudomonas aeruginosa quorumsensing regulons: effects of growth phase and environment. J Bacteriol 185, 2080-2095.

Wolfgang, M. C., Lee, V. T., Gilmore, M. E. \& Lory, S. (2003). Coordinate regulation of bacterial virulence genes by a novel adenylate cyclase-dependent signaling pathway. Dev Cell 4, 253263.

Wretlind, B. \& Pavlovskis, O. R. (1984). Genetic mapping and characterization of Pseudomonas aeruginosa mutants defective in the formation of extracellular proteins. J Bacteriol 158, 801-808.

Edited by: W. Bitter 